\title{
Energy transfer upconversion measurements for popular neodymium-doped crystals
}

\author{
SungJin Yoon*a, RengPen Yan ${ }^{\mathrm{b}}$, Stephen J. Beecher ${ }^{\mathrm{a}}$, Jacob I. Mackenzie ${ }^{\mathrm{a}}$ \\ ${ }^{a}$ Optoelectronics Research Centre, University of Southampton, Southampton SO17 1BJ, UK \\ ${ }^{b}$ National Key Laboratory of Science and Technology on Tunable Laser, Harbin Institute of \\ Technology, Harbin 150080, China
}

\begin{abstract}
We report our investigations on measuring the energy transfer upconversion (ETU) parameter in various neodymiumdoped laser crystals (YAG, $\mathrm{YVO}_{4}, \mathrm{GdVO}_{4}, \mathrm{KGW}$, and YLF) via the z-scan technique. Starting with a simple two-level macro-parameter spatially dependent rate equation model we obtain a good correlation for Nd:YAG at different concentrations and crystal temperatures, however the other crystals illustrate significant deviation between simulation and measurement. Currently we attribute this difference to additional ion-ion interactions in the respective samples, for which a more detailed model is currently being considered. Of the tested materials Nd:YAG appears to have the lowest ETU macro parameter, at around $0.35 \times 10^{-16} \mathrm{~cm}^{3} / \mathrm{s}$ for a 0.6 at.\% doping concentration, compared with nominally thrice this for 0.5 at\% Nd:YLF and almost an order of magnitude higher for the 0.5 at.\% vanadates $\left(\mathrm{YVO}_{4}\right.$ and $\left.\mathrm{GdVO}_{4}\right)$. These values are significant for determining additional heat load in the respective gain media, especially when trying to increase the output power/energy from lasers employing these crystals, typically achieved by increasing the pump and cavity mode size.
\end{abstract}

Keywords: Energy transfer upconversion, Nd:YAG, neodymium doped laser crystals, vanadate, upconversion

\section{INTRODUCTION}

Shortly after the invention of the laser, $\mathrm{Nd}^{3+}$ emerged as one of the most promising ions to incorporate into solid state media for laser applications [1]. This was due to several favorable attributes which make this ion very suitable for low, medium and high power applications. The first property is its broad absorption bands covering most of the visible spectrum with especially strong absorption between $700-900 \mathrm{~nm}$, well suited to pumping with krypton flash lamps, a property now largely redundant for most applications due to advances in high-power diode lasers. The second is the true 4-level nature of the $1 \mu \mathrm{m}$ emission band, reducing thresholds considerably in comparison to lasers exhibiting reabsorption losses. The third beneficial attribute is the very large emission cross section of the $1 \mu \mathrm{m}$ emission band, providing excellent gain characteristics leading to low excitation densities required to reach threshold, along with the relatively long lifetime of the upper level of this transition, making that population inversion easy to achieve. Despite the effective exploitation of a range of $\mathrm{Nd}^{3+}$ doped crystals the value of several parameters affecting laser performance remain either unreported or reported with such inconsistency between reports that the laser designer cannot make reliable choices for design parameters.

In this paper we will review some of the spectroscopic properties of five common laser crystals and present quantitative measurements of two detrimental non-radiative de-excitation mechanisms for these crystals, following these reports we will discuss their effect on laser performance both for continuous wave oscillators and for high inversion systems such as Q-switched oscillators.

\section{$1.1 \mathrm{Nd}^{3+}$ spectroscopy}

Figure 1. presents the first 9 energy level manifolds for trivalent neodymium with the commonly used transitions for Nd:YAG noted by their emission wavelength (the quasi-four-level 946nm transition, the four level 1064nm transition and the four level $1338 \mathrm{~nm}$ transition). The manifolds are spaced such that with the exception of the metastable ${ }^{4} \mathrm{~F}_{3 / 2}$ and ground state ${ }^{4} \mathrm{I}_{9 / 2}$ manifolds, multi-phonon decay rapidly de-excites ions from higher lying manifolds to these levels. Also illustrated in Figure 1., are two types of energy transfer mechanism: cross relaxation (CR), where an ion occupying 
the ${ }^{4} \mathrm{~F}_{3 / 2}$ manifold and an ion occupying the ${ }^{4} \mathrm{I}_{9 / 2}$ manifold 'share' their energy, transferring both ions into intermediate energy levels; and energy transfer upconversion (ETU), where two ions in the ${ }^{4} \mathrm{~F}_{3 / 2}$ manifold exchange energy to elevate one of these ions to a higher lying energy level while the other is demoted to a lower energy level. Due to the rapid multi-phonon decay a single CR event or a single ETU event can be thought of as reducing the ${ }^{4} \mathrm{~F}_{3 / 2}$ population by one and increasing the ${ }^{4} \mathrm{I}_{9 / 2}$ population by one.

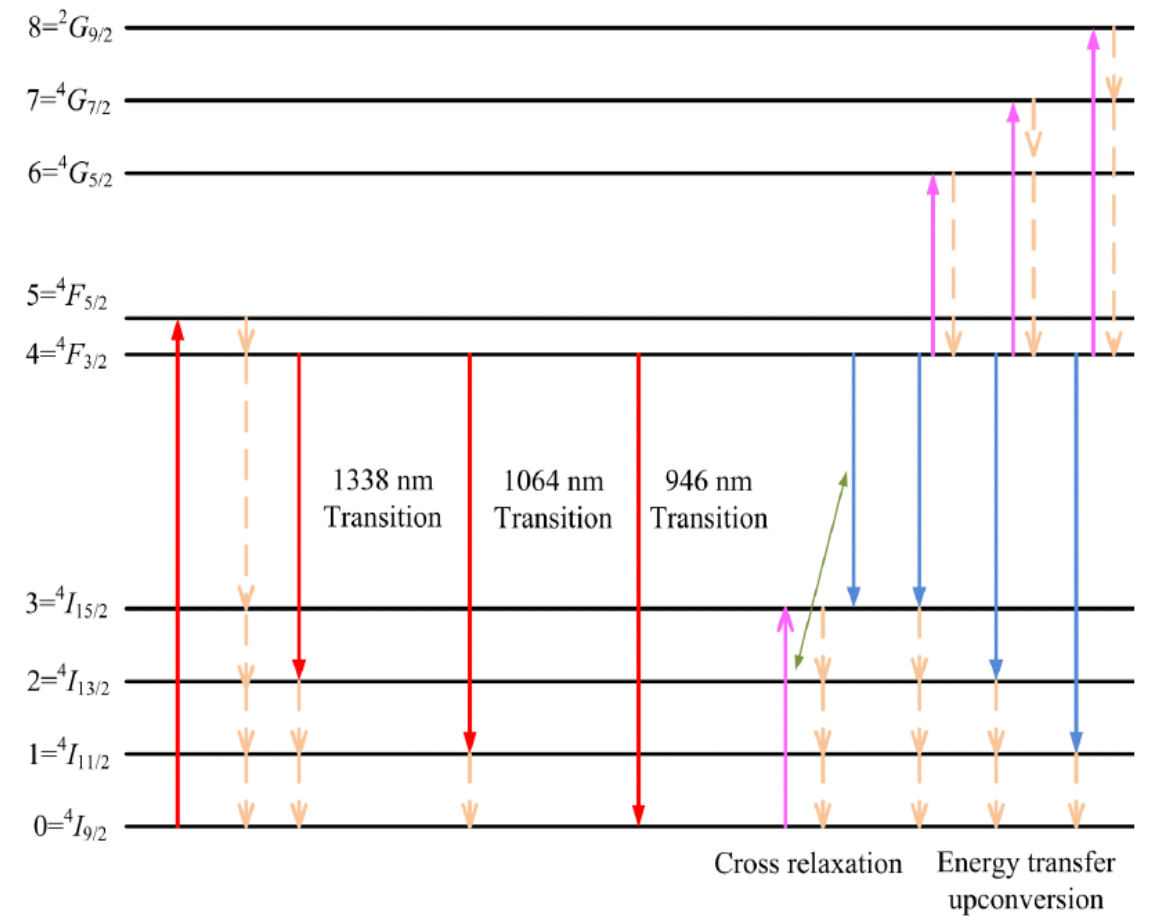

Figure 1. A simplified energy level diagram of Nd:YAG. Red arrows illustrate radiative and absorptive events (photonmediated) and dotted yellow arrows illustrate non-radiative events (phonon-mediated). Blue and Purple arrows illustrate inter-ionic energy transfer events including cross relaxation and energy transfer upconversion.

\section{METHODOLOGY}

Based upon these assumptions the system can be modelled by a simple spatially dependent two-level rate equation.

$$
\begin{gathered}
\frac{\partial N_{1}(r, z)}{\partial t}=-\frac{I_{P}(r, z)}{h v_{P}} \sigma_{a b s} N_{1}(r, z)+\frac{N_{2}(r, z)}{\tau_{0}}+W_{E T U} N_{2}(r, z)^{2}+W_{C R} N_{1}(r, z) N_{2}(r, z) \\
\frac{\partial N_{2}(r, z)}{\partial t}=\frac{I_{P}(r, z)}{h v_{P}} \sigma_{a b s} N_{1}(r, z)-\frac{N_{2}(r, z)}{\tau_{0}}-W_{E T U} N_{2}(r, z)^{2}-W_{C R} N_{1}(r, z) N_{2}(r, z), \\
\frac{d I_{P}(r, z)}{d z}=I_{P}(r, z)\left(-\sigma_{a b s} N_{1}(r, z)\right) .
\end{gathered}
$$

where $\tau_{0}$ is the intrinsic radiative lifetime for the $\mathrm{Nd}^{3+}$ doped crystal, $\sigma_{a b s}$ is the effective absorption cross section for the ground state level $N_{1}, h v_{P}$ is the pump photon energy, $W_{E T U}$ is the ETU coefficient macro-parameter, $W_{C R}$ is the CR coefficient macro-parameter, and $I_{P}(r, z)$ is the pump irradiance distribution along the sample's length.

To avoid the need for ray-tracing we work with an effective beam area $A_{\text {eff }}$ obtained from the average beam radius over the crystal length using Gaussian beam propagation theory.

$$
A_{\text {eff }}=\frac{\pi}{l_{c}} \int_{0}^{l_{c}} \omega(z)^{2} \mathrm{~d} z .
$$


More extensive details of the model and its application to Nd:YAG are available in [2].

To be able to calculate the ETU coefficient with any degree of confidence the other parameters within equations 1-4 need to be known to a high degree of accuracy. We conducted a detailed spectroscopic characterization of the different crystals studied, using a setup similar to that described in [3], in addition to including a polarizer, placed after the crystal, to allow for the absorption cross sections for the different optical axes of the anisotropic crystals to be measured.

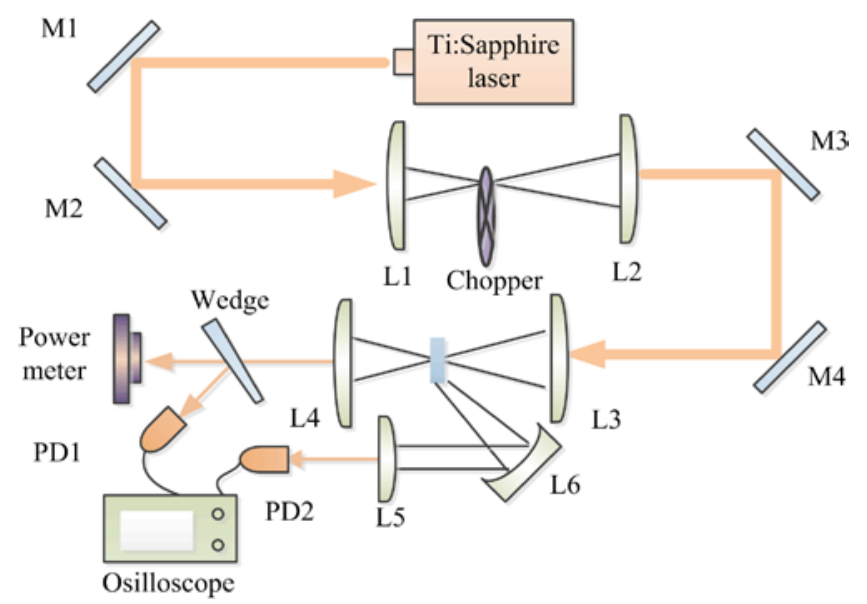

Figure 2. Experimental setup for the Z-scan measurements. A tunable CW Ti:Sapphire laser beam is mechanically chopped to reduce the average power before being focused through a crystal. L3 and L4 are scanned to change the beam radius in the crystal and hence the irradiance of the beam in the crystal. The transmitted power, the temporal dependence of the transmitted power and the temporal dependence of the fluorescence are captured for comparison with a numerical model.

The ETU coefficients are measured using an adaptation of the open aperture Z-scan technique [4]. This technique has previously been employed to measure ETU coefficients, initially of Nd doped materials by Jacinto et al. [5], and more recently of Er:YAG by White et al. [6]. At low irradiances absorption of the pump light follows Beers law but at irradiance levels approaching the saturation irradiance of the gain medium a significant depletion of the ground $\left({ }^{4} \mathrm{I}_{9 / 2}\right)$ state occurs. This depletion of the ground state leaves fewer ions in the pump path capable of absorbing the pump radiation resulting in an increase in transmission. ETU acts as an additional de-excitation mechanism, in addition to nonradiative decay (NR-decay), spontaneous emission and cross relaxation, where we neglect stimulated emission since we use a short crystal length in a low Q cavity (i.e. feedback equivalent to Fresnel reflections from the crystal facets). Since the rates of NR-decay, spontaneous emission and cross relaxation can be established with high accuracy at low irradiance, deviations from a simple model of these processes at high irradiance can be attributed uniquely to ETU, which allows a simple and accurate measurement of its macro-parameter coefficient. Table 1 presents the key spectroscopic properties measured for the investigated crystals.

Table 1. Key spectroscopic properties of the investigated laser crystals.

\begin{tabular}{|c|c|c|c|c|c|}
\hline Host & Lifetime $(\mu \mathrm{s})$ & $\sigma_{\text {abs }}\left(\mathrm{pm}^{2}\right)$ & $\begin{array}{l}\text { Ions per } \mathrm{cm}^{3} \text { per at. } \% \\
\left(\mathrm{x} 10^{20}\right)\end{array}$ & $\begin{array}{l}\text { Nominal dopant } \\
\text { concentration } \\
\text { (at.\%) }\end{array}$ & Length (mm) \\
\hline YAG & 260 & 6.8 & 1.38 & $\begin{array}{l}0.3 \\
0.6 \\
1 \\
1.1\end{array}$ & $\begin{array}{l}5 \\
1 \\
3.25 \\
1\end{array}$ \\
\hline YLF & 520 & 11 & 1.32 & 0.5 & 1 \\
\hline $\mathrm{YVO}_{4}$ & $\begin{array}{l}98 \\
90\end{array}$ & 60 & 1.25 & $\begin{array}{l}0.5 \\
1\end{array}$ & $\begin{array}{l}1 \\
1\end{array}$ \\
\hline $\mathrm{GdVO}_{4}$ & $\begin{array}{l}107 \\
98\end{array}$ & 50 & 1.21 & $\begin{array}{l}0.5 \\
1\end{array}$ & $\begin{array}{l}1 \\
1\end{array}$ \\
\hline KGW & $\begin{array}{l}112 \\
112\end{array}$ & 26 & 0.63 & $\begin{array}{l}2 \\
4\end{array}$ & $\begin{array}{l}1 \\
1\end{array}$ \\
\hline
\end{tabular}




\section{RESULTS}

\subsection{Nd:YAG}

We have previously published values for the ETU coefficients of 1.0 at.\% Nd:YAG as a function of temperature [2]. Where it was shown that the ETU coefficient decreased with increasing temperature, following a trend of $\mathrm{W}_{\mathrm{ETU}}=13.3-$ $0.037 \mathrm{~T}+2.4 \times 10^{-6} \mathrm{~T}^{2}$. This decreasing behavior coupled to the decreasing peak absorption and emission cross sections leads to a complicated interplay between the thermal loading of the crystal and laser threshold for CW systems. This effect is further complicated when a pump source with a significant bandwidth, e.g. typical high-power diode lasers, where the effective absorption coefficient can actually increase with increasing temperature due to broadening of the Nd:YAG absorption peak [2]. Further analysis on the combination of these effects in typical diode end-pumped Nd:YAG lasers will be published shortly. Figure 3., presents our data for the ETU rate dependence on the neodymium dopant concentration in YAG. In our analysis we have assumed that the intrinsic lifetime of the ${ }^{4} \mathrm{~F}_{3 / 2}$ energy level is $260 \mu \mathrm{s}$ and that measured deviations in the fluorescence lifetime at low excitation density are due entirely to CR. This allows us to calculate a CR macro-parameter for each crystal to fit the measured fluorescence lifetimes of 253, 250, 235 and $232 \mu \mathrm{s}$ for $0.3,0.6,1.0$ and 1.1 at.\% respectively. The best fit $\mathrm{W}_{\mathrm{ETU}}$ parameters show that the rate of ETU increases with increasing dopant concentration. Good agreement is observed between the transmitted waveform and the model giving us confidence in these results

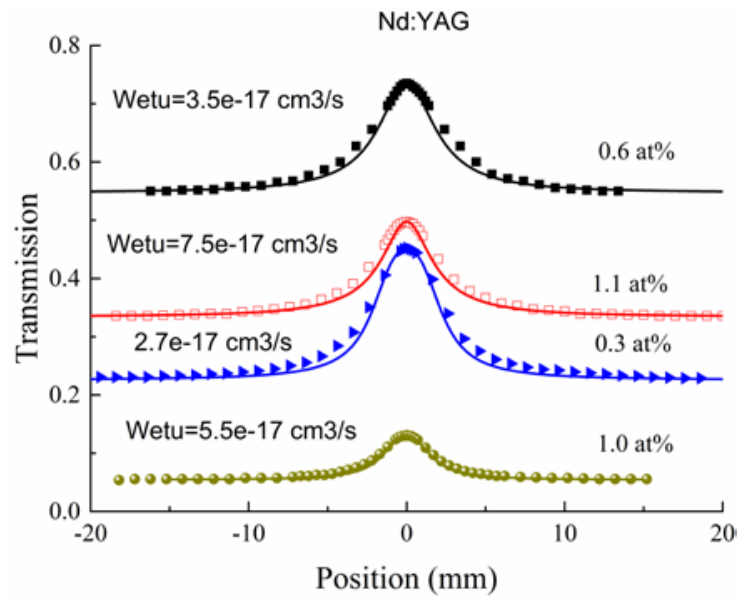

Figure 3. Z-scan trace for the different Nd:YAG crystals tested, i.e. pump transmission through the laser crystal for different positions of the beam waist relative to its center. The only free parameter used within the fitting is $\mathrm{W}_{\mathrm{ETU}}$.

\subsection{Nd:YLF}

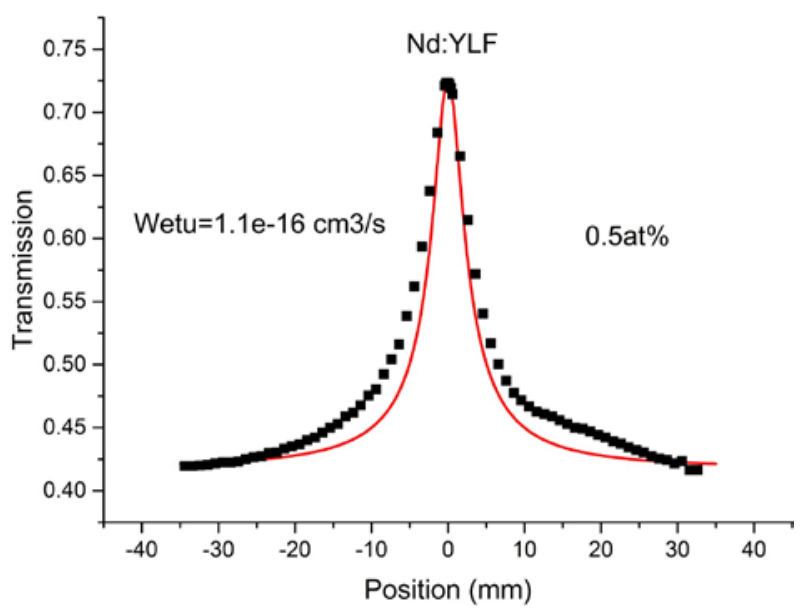

Figure 4. Z-scan trace for 0.5 at.\% doped Nd:YLF. 
For Nd:YLF (and the remainder of the crystals) we adopted a different procedure. Here the measured fluorescence lifetime of each crystal is assumed to be the intrinsic lifetime and the role of cross-relaxation is neglected. The fit for $\mathrm{Nd}$ :YLF is not as good as that obtained for Nd:YAG reducing our confidence in this result, nonetheless the value is in agreement with the trend seen by comparing Jacinto's and Pollnau's measurements [5,7]. It is possible that some processes are occurring that the model is not capable of capturing. There is no doubt however that the transmission of the pump beam through the sample when the crystal is at focus is significantly lower than would be expected if no nonlinear lifetime quenching of the ${ }^{4} \mathrm{~F}_{3 / 2}$ level or excited state absorption were occurring. Our modelled $\mathrm{W}_{\mathrm{ETU}}$ value is significantly higher than that of Nd:YAG.

\subsection{Nd:YVO4}

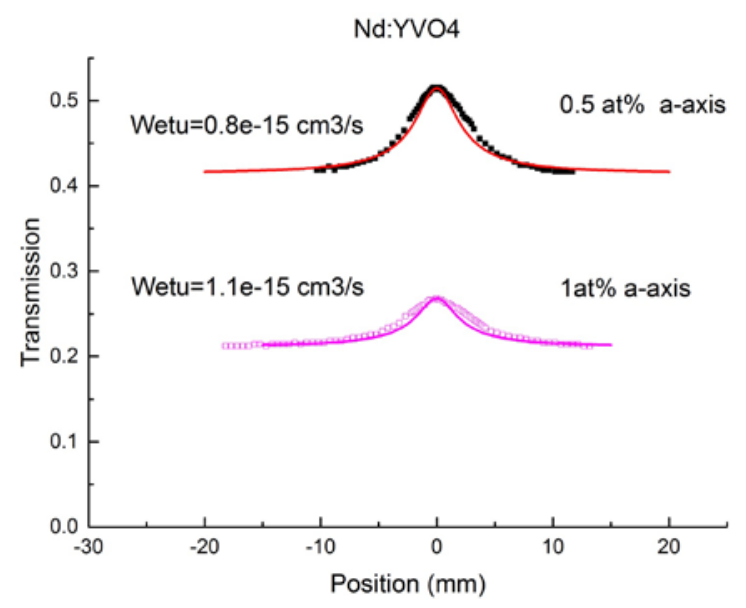

Figure 5. Z-scan trace for $\mathrm{Nd}: \mathrm{YVO}_{4}$ for a polarized pump (E\|a-axis).

The fit for Nd: $\mathrm{YVO}_{4}$ appears reasonable and the temporal waveforms match well with the model. The retrieved $\mathrm{W}_{\mathrm{ETU}}$ parameter for $\mathrm{Nd}: \mathrm{YVO}_{4}$ is very large, approximately two orders of magnitude higher than for Nd:YAG. For CW cases this can be partially offset by the larger emission cross section but is still likely to become a significant depopulation mechanism in some laser architectures in particular those with short gain media (microchip lasers) where a high inversion density may be required to reach threshold.

\subsection{Nd:GdVO4}
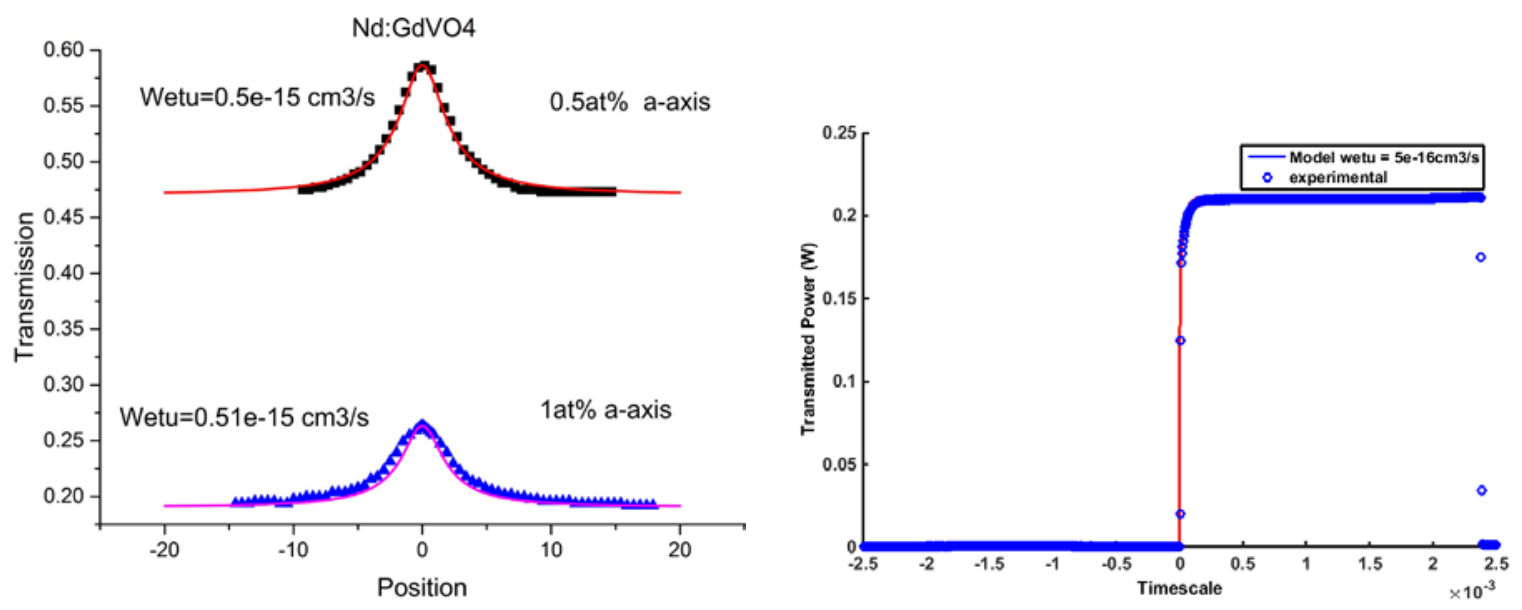

Figure 6. (Left) Z-scan trace for $\mathrm{Nd}: \mathrm{GdVO}_{4}$ and (Right) the temporal dependence of the transmission of the polarized pump (E\|a-axis) through the laser crystal for the 0.5 at.\% crystal at the position of highest transmission (the focus).

The fit for $\mathrm{Nd}: \mathrm{GdVO}_{4}$ is good at $0.5 \mathrm{at} . \%$ and only degrades slightly for the 1at.\% sample. We believe this degradation of the fit to be predominantly down to lack of signal strength due to the very strong pump absorption in the $1 \mathrm{~mm}$ long 
crystal. The temporal transmission of the pump is fitted very well by the simple model giving us confidence in our $\mathrm{W}_{\mathrm{ETU}}$ value, again nominally two orders of magnitude larger than for Nd:YAG.

\subsection{Nd:KGW}

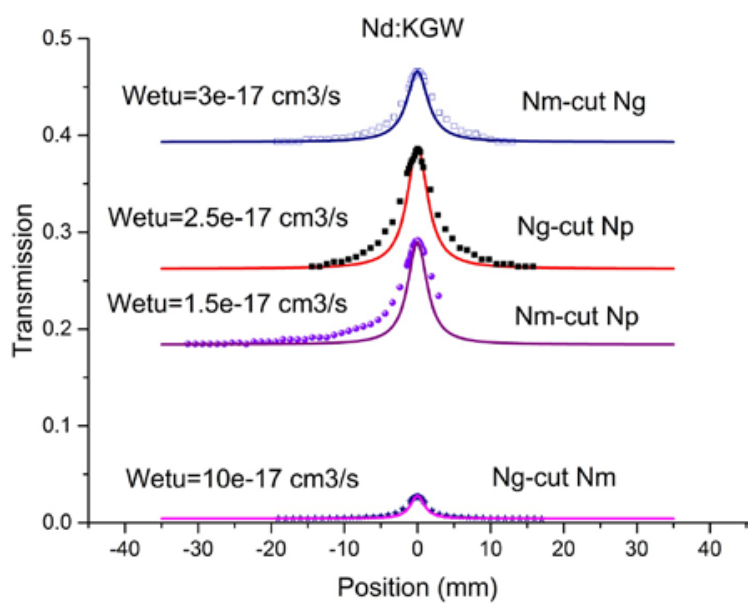

Figure 7. Z-scan trace for 2 at.\% ( $\mathrm{N}_{\mathrm{m}}$ cut) and 4 at.\% ( $\mathrm{N}_{\mathrm{g}}$ cut) doped $\mathrm{Nd}$ :KGW crystals , with the pump polarized along different principal axes, (i.e. $\mathrm{E}\|\mathrm{Ng}, \mathrm{E}\| \mathrm{Np}$, or $\mathrm{E} \| \mathrm{Nm})$.

For Nd:KGW the fit is relatively poor, which we believe could be due to excited state absorption of the pump light from the ${ }^{4} \mathrm{~F}_{3 / 9}$ energy level. Our confidence in these results is not high, since processes not included in the model are clearly occurring. That said the actual magnitude of the sum of all of these detrimental processes appears to be low, and the transmitted pump power when the crystal is at focus is not far below that predicted in the absence of any non-linear depopulation mechanism. This result is also evidenced by the absence of any reduction in the measured florescence lifetime when the dopant concentration in increased from 2 at.\% to 4 at.\%. We can cautiously state that we believe the magnitude of the sum of the nonlinear depopulation mechanisms may even be below that of Nd:YAG.

\subsection{Summary of results}

Here we present a summary of the ETU coefficients for the different crystals. We have included plots with the abscissa denoting both the dopant concentration in at.\% and in ions per $\mathrm{cm}^{3}$. While at.\% is more commonly used to describe crystals in research papers and by manufacturers we feel that ions per $\mathrm{cm}^{3}$ allows for a better comparison of the relative transfer rates within the different hosts. Again it should be noted that the values given for KGW are likely inaccurate and we believe the energy dynamics within this crystal are more complicated than allowed for in the model used.
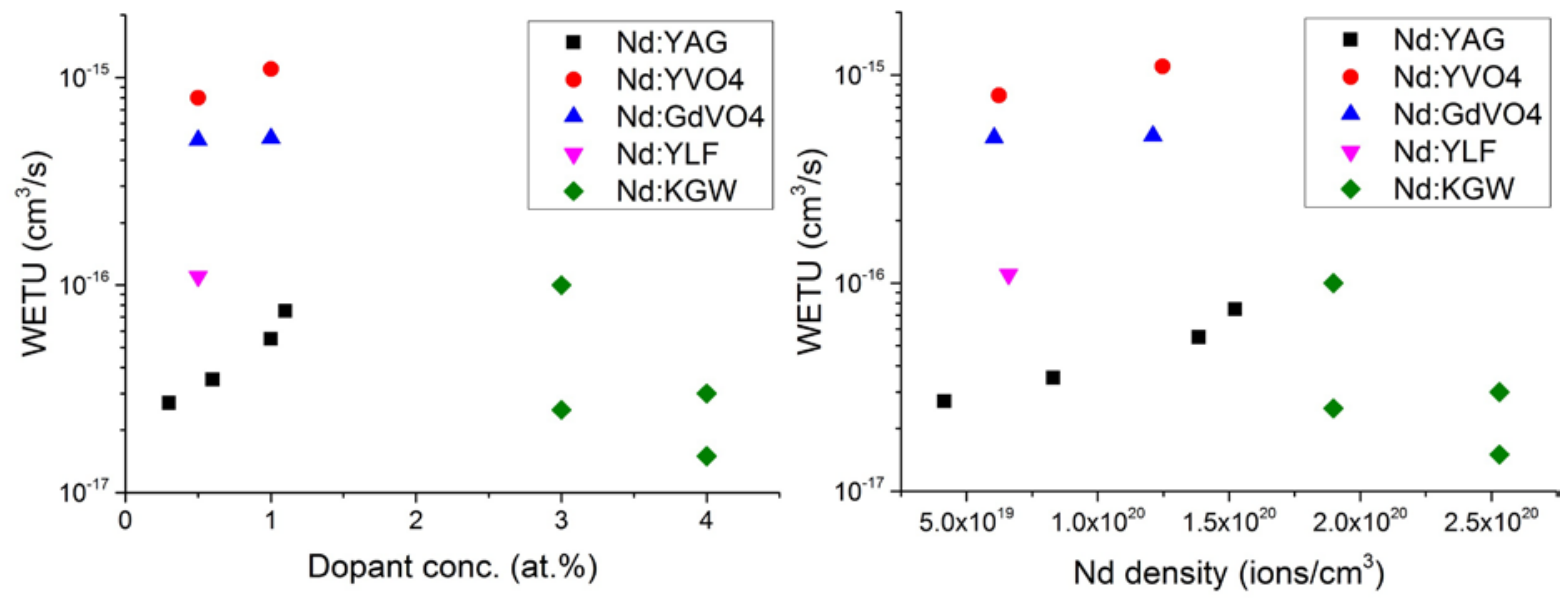

Figure 8. ETU parameter as a function of (Left) atomic dopant concentration and (Right) ion density for the crystals tested. For Nd:KGW the two data points for each crystal illustrate the values obtained for the different polarizations accessible in our samples the spread between these values should be viewed as a minimum uncertainty. 


\section{DISCUSSION}

\subsection{Effects on CW laser performance}

For continuous wave oscillators the dominant effect of ETU is an increase in laser threshold caused by the effective decrease in upper state lifetime with increasing excitation density. Since the average inversion density is clamped at laser threshold, only small changes based on the distribution of the inversion density are expected at higher pump powers. For this reason for the simple analysis described herein these effects are neglected. We base our analysis on the work of Kim et al. [8], who provide an analytic expression for the modification of the laser threshold in a gain media due to ETU effects for end pumped CW lasers. We assume that the crystals are three absorption lengths long so that near complete pump absorption occurs, and that the pump focusing is arranged such that pump-beams' confocal parameter in the crystal is set equal to the crystal length. We use an effective absorption length taken for a typical $100 \mu \mathrm{m}, 0.22 \mathrm{NA}$ fiber coupled laser diode with a FWHM bandwidth of $3.5 \mathrm{~nm}$. In addition we set a mode-overlap factor [9] equal to 1.2, this has been numerically shown to maximize slope efficiencies for Gaussian laser modes end pumped by 'top hat' type pump beams. Under these conditions, laser threshold for a variety of output couplers can be calculated. It should be noted that due to the fast multi-phonon decay from the energy levels populated by ETU to the ${ }^{4} \mathrm{~F}_{3 / 2}$ and ${ }^{4} \mathrm{I}_{9 / 2}$ any additional pump power required to reach laser threshold due to ETU is deposited as heat in the gain medium and that this occurs predominantly at the pump input face where the inversion density is greatest. In all cases we have assumed 1\% parasitic cavity losses.

$$
P_{t h} \approx \frac{h v_{p} \pi \omega_{p}^{2}}{2\left(f_{1}+f_{2}\right) \sigma \tau \eta_{q} \eta_{L P}}\left(L_{T}+2 f_{1} \sigma \eta_{L P} N_{t} l_{R}\right)\left[1+\frac{W_{u p} \tau \alpha_{p}}{4\left(f_{1}+f_{2}\right) \sigma \eta_{L P}}\left(L_{T}+2 \eta_{L P} f_{1} \sigma N_{t} l_{R}\right)\right]
$$

For details of the derivation of the threshold equation in the presence of ETU readers are directed to [6], briefly however the part outside of the square brackets is a standard expression for laser threshold in the presence of reabsorption losses but in the absence of ETU, while the part inside the square brackets is a modification to account for these effects.

\subsubsection{Nd:YAG}

For the four different dopant concentrations of Nd:YAG trialled here, ETU shows no significant effect on laser threshold for the $1064 \mathrm{~nm}$ laser transition. Calculated pump radii were $510 \mu \mathrm{m}, 360 \mu \mathrm{m}, 280 \mu \mathrm{m}$ and $265 \mu \mathrm{m}$ for 0.3, 0.6, 1.0 and 1.1 at.\% doped crystal with lengths of $42.4 \mathrm{~mm}, 21.2 \mathrm{~mm}, 12.7 \mathrm{~mm}$, and $11.6 \mathrm{~mm}$, respectively. Pump absorption coefficients were calculated using the room temperature case of the equation given by Yoon et al. [3].

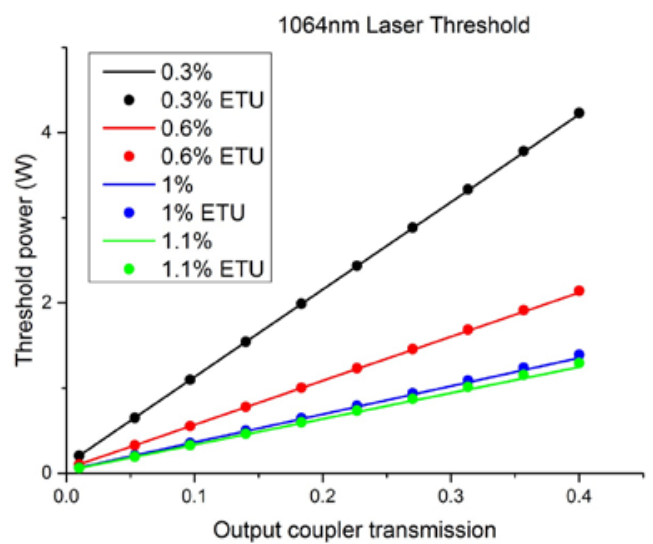

Figure 9. The effect of energy transfer upconversion on laser threshold of 1064nm Nd:YAG CW lasers. Over the modelled parameter space ETU has no significant effect.

For the 946 nm quasi-four-level transition the $\sim 7 x$ smaller emission cross section, which also suffers a weak reabsorption loss, has a significantly higher laser threshold, exacerbated by the effect of ETU that becomes quite significant at modest output coupling values. This implies that total cavity losses must be kept low, allowing for low output coupling, in order for this transition to be efficient and to avoid significant additional heat load due to ETU at the input face of the crystal. 


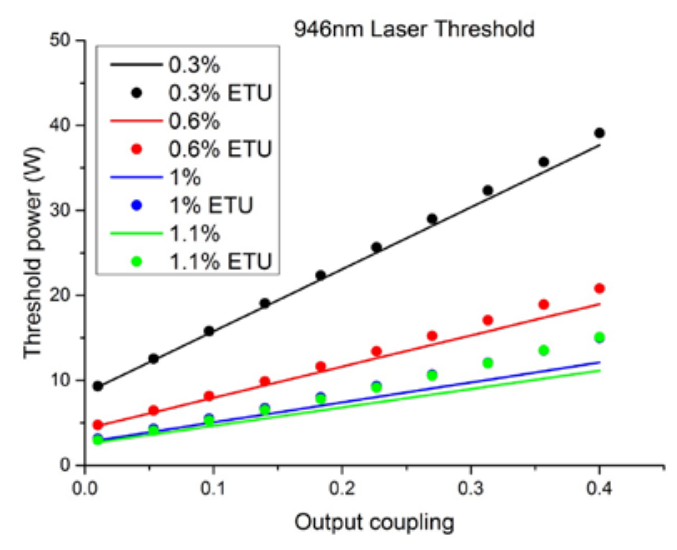

Figure 10. The effect of energy transfer upconversion on laser threshold for 946nm Nd:YAG CW lasers. ETU has a significant effect for output coupling transmission above $\sim 15 \%$ for the 1.0 and 1.1 at.\% crystal but a far lesser effect for the 0.6 at. $\%$ and 0.3 at.\% crystals, while the pump irradiance dictates the higher threshold associated with these lowconcentration crystals.

\subsubsection{Nd:YVO}

The very strong absorption of Nd:YVO 4 allows for short crystals to be used if pumped with a polarised source. For our assumed pump source, beam radii of $41 \mu \mathrm{m}$ and $29 \mu \mathrm{m}$ can be used for the 0.5 at.\% and 1.0 at.\% doped crystals of lengths $3.0 \mathrm{~mm}$ and $1.5 \mathrm{~mm}$ respectively leading to very low thresholds. In this case the high inversion density and the more than order of magnitude higher ETU coefficient than Nd:YAG lead to a large increase in the laser threshold for output coupling above $\sim 10 \%$.

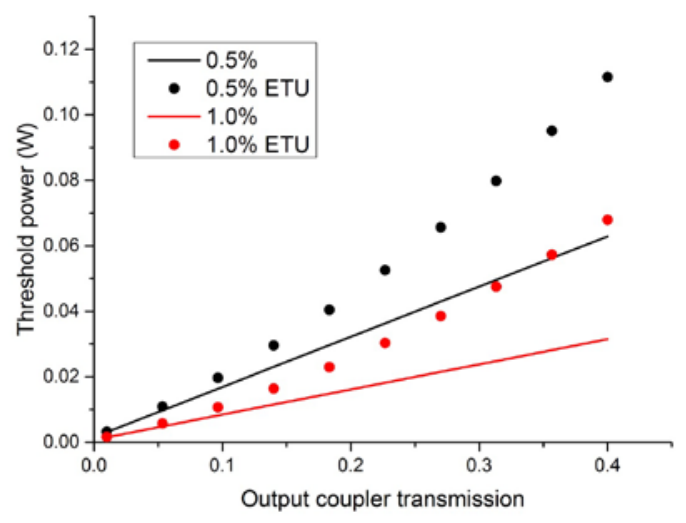

Figure 11. Strong effects of energy transfer upconversion are seen on laser threshold for $1064 \mathrm{~nm} \mathrm{Nd:YVO}$. Even output coupling of $10 \%$ will result in a significant increase in heat load in comparison to the hypothetical case of zero ETU.

\subsection{Effects on pulsed laser performance}

For pulsed systems (and high gain small signal amplifiers) we follow a different methodology. In this case, for simplicity, we assume a uniformly inverted gain medium, a good approximation of a well-designed flashlamp pumped system or diode side pumped system as is often favored for high energy, low repetition rate Q-switched systems. To allow for a sensible comparison between different gain media we have plotted effective lifetime vs gain per unit length for these media and compared on the same graph. Effective life times are calculated using an analytical solution to the Bernoulli equation, here we neglect the cross-relaxation component and assume that the lifetime of the ${ }^{4} \mathrm{~F}_{3 / 2}$ level is the measured fluorescence lifetime of the crystal. Details of the application of this equations to measuring ETU parameters can be found in [10]. 


\subsection{1 ${ }^{4} \mathbf{I}_{11 / 2}$ terminating emission (the $1064 \mathrm{~nm}$ line of Nd:YAG)}

These results show that, as is well known in the field, while the energy storage of YLF is better than any of the other crystals studied, this benefit reduces slightly when high gain per unit length is required (e.g. for short Q-switched pulses). For the $1 \mu \mathrm{m}$ case no real surprises are observed, with $\mathrm{Nd}: \mathrm{YVO}_{4}$ excelling for high gain per unit length, and $\mathrm{Nd}$ :YLF excelling for energy storage in applications where moderate gain per unit length can be tolerated.
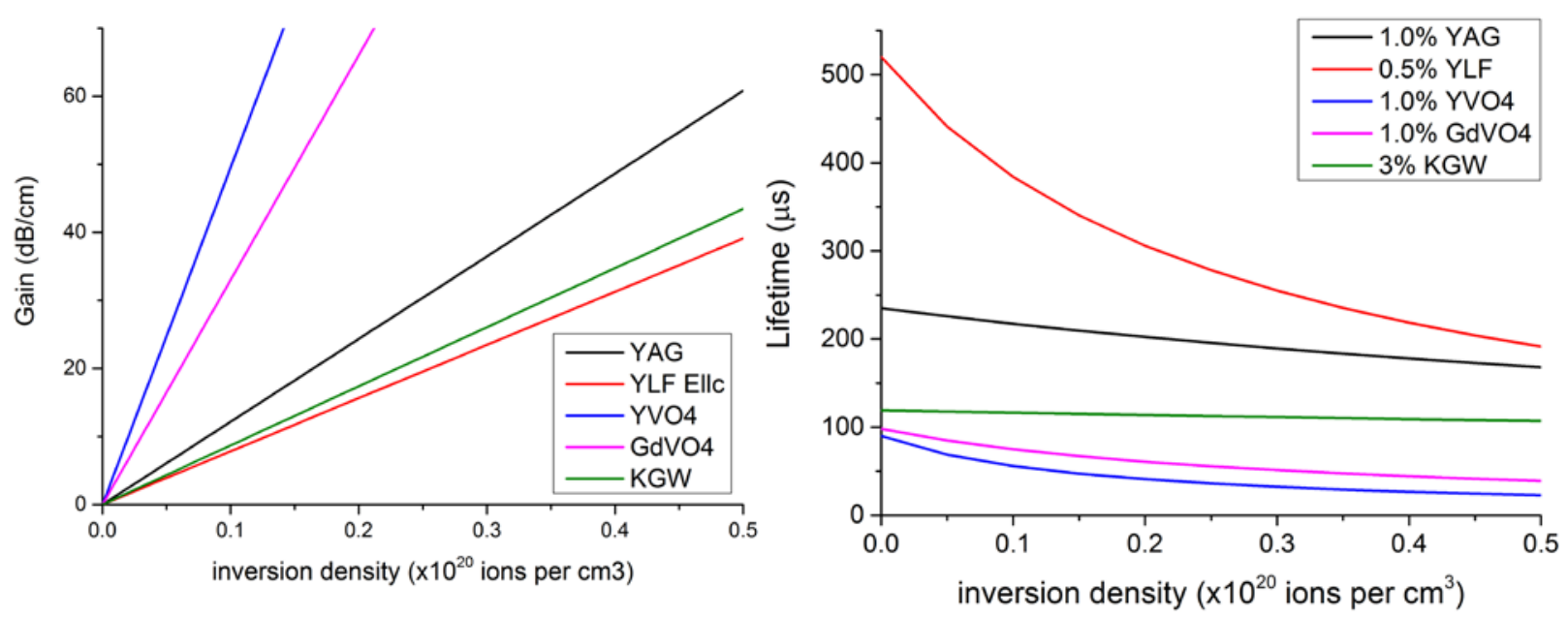

Figure 12. (Left) gain per unit length in function of the $\mathrm{Nd}^{3+}$ inversion density for the different crystals tested; (Right) the effective lifetime for these crystals at these inversion densities.

\subsection{2 ${ }^{4} \mathbf{I}_{9 / 2}$ terminating emission (the $946 \mathrm{~nm}$ line of Nd:YAG)}

For the case of the quasi-four-level 9xx nm transition reabsorption losses require a certain inversion density to reach transparency. This coupled to the significantly lower emission cross section results in ETU being far more significant. Taking YLF as a worst case example, in order to reach $2 \mathrm{~dB} / \mathrm{cm}$ gain, the inversion density required would reduce the upper state lifetime to close to that of YAG. These plots also highlight the significant advantages of $\mathrm{GdVO}_{4}$ over $\mathrm{YVO}_{4}$ for this transition.
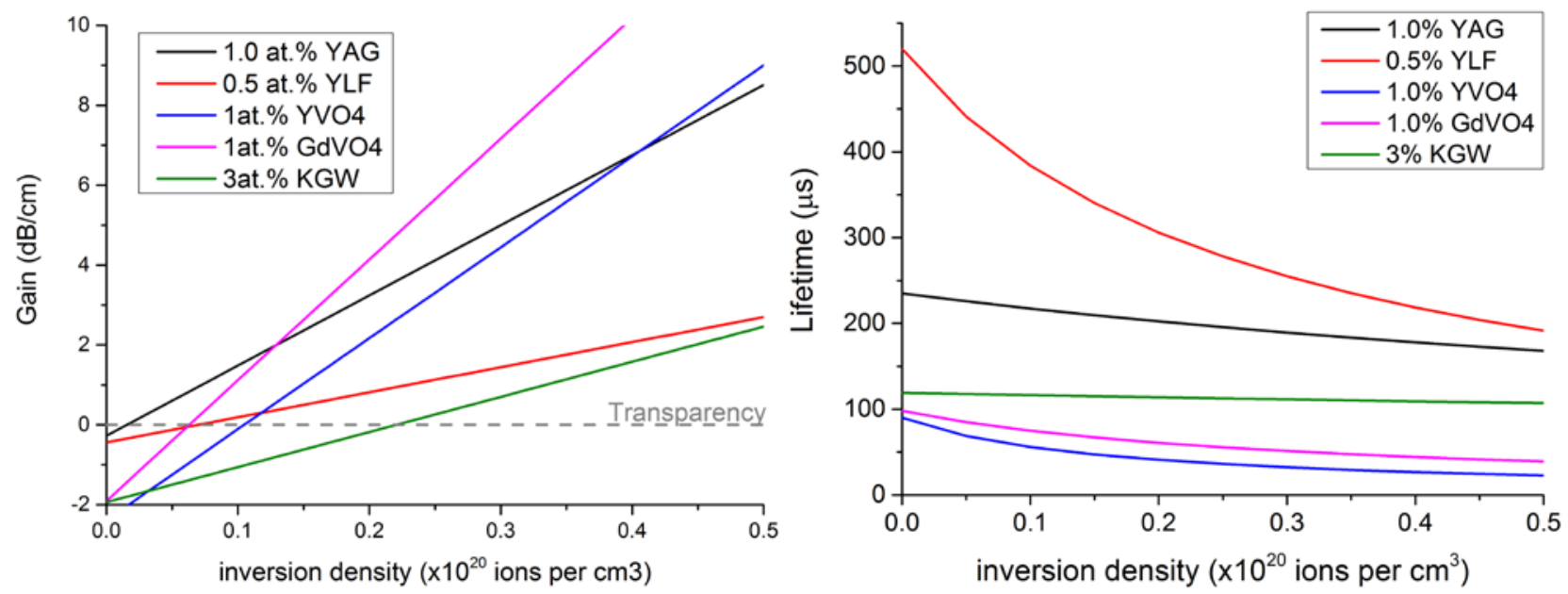

Figure 13. (Left) strongest $9 \mathrm{xx} \mathrm{nm}$ transition gain per unit length in function of the $\mathrm{Nd}^{3+}$ inversion density for the different crystals tested; (Right) the effective lifetime for these crystals at these inversion densities. 


\section{CONCLUSIONS}

We have demonstrated the utilization of the Z-scan technique to rapidly assess the impact of detrimental energy transfer effects on different neodymium doped laser crystals. Our results once again confirm how well suited YAG is as a host for $\mathrm{Nd}^{3+}$ and that its prevalence in the field of low, medium and high power lasers is not just a result of the superb thermal, mechanical and simple spectroscopic properties but also the crystals resistance to ETU. We have reported the effects that ETU is likely to have on two different laser operation modes, CW oscillators and highly inverted media such as high gain amplifiers or Q-switched lasers. The result of this analysis is that ETU has minimal effects for most CW systems operating on the dominant 4 level transition at $1 \mu \mathrm{m}$ but becomes significant for the quasi-four-level 9xx nm transition and for systems with high population inversions such as low repetition rate Q-switched lasers. For YLF and KGW some further work is required to fully assess why discrepancies between the results of the experiment and the model occur. Within the framework of the model we have only allowed ourselves one free fitting parameter $\left(\mathrm{W}_{\text {ETU }}\right)$ and to reconcile these discrepancies the introduction of further parameters for other processes will be necessary. The results presented are, we believe, the most comprehensive report of these detrimental processes in $\mathrm{Nd}^{3+}$ doped laser media to date.

\section{REFERENCES}

[1] Geusic, J. E., Marcos, H. M. and Van Uitert L. G., "Laser oscilations in Nd-doped yttrium aluminium, yttrium gallium and gadolinium garnets,” Appl. Phys. Lett. 4, 182-184 (1964).

[2] Yan, R. P., Yoon, S. J., Beecher, S. J. and Mackenzie, J. I., "Measuring the elevated temperature dependence of upconversion in Nd:YAG,” IEEE J. Sel. Top. Quantum Electron. 21(1), 1601208 (2015).

[3] Yoon, S. J. and Mackenzie, J. I., "Cryogenically cooled 946nm Nd:YAG laser," Opt. Express 22, 8069-8075 (2014).

[4] Sheik-bahae, M., Said, A. A. and Van Stryland, E. W., "High-sensitivity, single-beam $\mathrm{n}_{2}$ measurements," Opt. Lett. 14, 955-957 (1989).

[5] Jacinto, C., Messias, D. N., Andrade, A. A. and Catunda, T., "Energy transfer upconversion determination by thermal-lens and Z-scan techniques in $\mathrm{Nd}^{3+}$-doped laser materials," J. Opt. Soc. Am. B 26, 1002-1007 (2009)

[6] White, J. O. and Mungan, C. E., "Measurement of upconversion in Er:YAG via z-scan," J. Opt. Soc. Am. B 28, 2358-2361 (2011)

[7] Pollnau, M., Hardman, P. J., Kern, M. A., Clarkson, W. A. and Hanna, D. C., "Upconversion-induced heat generation and thermal lensing in Nd:YLF and Nd:YAG,” Phys. Rev. B 58, 16076 (1998)

[8] Kim, J. W., Mackenzie, J. I. and Clarkson, W. A., "Influence of energy-transfer-upconversion on threshold pump power in quasi-three-level solid-state lasers,” Opt. Express 17, 11935-11943 (2009).

[9] Taira, T., Tulloch, W. M. and Byer, R. L., "Modeling of quasi-three-level lasers and operation of cw Yb:YAG," Appl. Opt. 36, 1867-1874 (1997).

[10] Guyot, Y., Manaa, H., Rivoire, J. Y., Moncorge, R., Gamier, N., Descroix, E., Bon, M. and Laporte, P., "Excitedstate-absorption and upconversion studies of $\mathrm{Nd}^{3+}$-doped single crystals $\mathrm{Y}_{3} \mathrm{~A}_{15} \mathrm{O}_{12}, \mathrm{YLiF}_{4}$, and $\mathrm{LaMgAl}_{11} \mathrm{O}_{19}$," Phys. Rev. B 51(2), 784-799 (1995). 